\title{
A case of Brucella melitensis endocarditis in a patient with cardiovascular implantable electronic device
}

\author{
Wasl Al-Adsani \\ Ali Ahmad \\ Mohammad Al-Mousa \\ Department of Internal Medicine, \\ Mubarak Al-Kabeer Hospital, Jabriya, \\ Kuwait
}

This article was published in the following Dove Press journal: Infection and Drug Resistance

Background: Brucellosis is a bacterial infection, caused by the nonmotile gram-negative aerobic coccobacilli. The clinical manifestation of brucellosis is variable, ranging from mild disease to severe disease. The gold standard test to confirm the diagnosis of brucellosis is assaying blood, bone marrow, or other body fluids or by tissue culture. Preferred first-line treatment in adults with uncomplicated, nonlocalized disease consists of doxycycline-aminoglycoside combination. The recommended duration of treatment is 6 weeks. Cardiovascular implantable electronic device (CIED) infection caused by brucellosis is rare and has been reported in a few case study series. We report a case of Brucella melitensis infection of CIED where, contrary to most authorities recommending removal of device, because of the patient's multiple comorbid conditions and age, an attempt was made to keep the device and place the patient on lifelong prophylaxis treatment.

Case report: A 70-year-old Kuwaiti male, with a background history of Type 2 diabetes mellitus, atrial fibrillation, hypertension, and sick sinus syndrome with a pacemaker fixed 1 year prior to his presentation, was diagnosed as having brucellosis. He was initially started on rifampicin and doxycycline. A transesophageal echo showed no evidence of vegetations seen at aortic valve or mitral valve. The two pacing leads, one in right atrium appendage and the other in right valve apex, looked smooth and clean with no clear adherence. Cotrimoxazole and gentamicin were added, per the 2015 European Society of Cardiology Guidelines for the management of infective endocarditis. The plan was to treat the patient with an aminoglycoside for the first 3 weeks, and then de-escalate to three drugs. The patient's fever subsided for several days following treatment, and subsequent blood cultures showed sterilization of bloodstream. He developed an acute kidney injury from aminoglycosides, which required 2 months of inpatient dialysis treatment. He was later discharged after completing a total of 3 months of antibiotic treatment. At the time of discharge, the patient's Brucella titer was $<1: 160$. The patient was discharged with a 2-month supply of rifampin and doxycycline.

Conclusion: In all published studies on Brucella infective endocarditis with a cardiovascular implantable device, the recommendation was for device removal and extended treatment with doxycycline combined with rifampin and/or trimethoprim-sulfamethoxazole, plus parenteral aminoglycosides for the first 3 weeks. There have been two cases in the literature that have studied CIED infected with brucellosis where an attempt was made to retain the device. In both cases, relapse occurred upon discontinuation of treatment. It is not clear whether lifelong antibiotic suppression treatment would prevent relapse.

Keywords: brucellosis melitensis, CIED infection, ELISA, brucellosis endocarditis, Brucella melitensis endocarditis
Derrespondence: Ali Ahmad Mubarak Al-Kabeer Hospital, Block 4, Street 103, Jabriya, Kuwait 46300 Email aahmad@tcd.ie 


\section{Background}

Brucellosis is a bacterial infection associated with contact of fluids from infected animals (goats, sheep, camels, dogs) or one that arises due to consumption of unpasteurized milk and cheese. The estimated incidence of brucellosis worldwide ranges from 0.03 to 160 per $100,000 .^{1}$

The disease is mostly seen in the Mediterranean Basin, Arabian Peninsula, Indian subcontinent, central and South America, the Balkans, and parts of the former Soviet Union., ${ }^{2,3}$

Brucella is a nonmotile intracellular gram-negative aerobic coccobacillus. Several species cause human disease, including Brucella melitensis, which is also associated with infections in goats and camels; Brucella canis, associated with dogs; Brucella suis associated with pigs; and Brucella abortus associated with cattle. Most human disease is caused by B. melitensis and B. abortus. In the developing world, transmission is commonly due to ingestion of contaminated milk from goats, sheep, or camels. Other forms of transmission occur through direct contact on handling an infected animal, inadvertent inoculation through skin cuts, conjunctival inoculation, or inhalation of infected aerosols..$^{2,3}$

The clinical manifestation of brucellosis is variable, ranging from mild disease to severe disease. Similar to syphilis, brucellosis has many of clinical manifestations, and is hence likened as a "Great Imitator". ${ }^{1}$ The incubation period is typically 1-4 weeks. However, the onset of symptoms varies; it can manifest as acute febrile illness, relapsing/undulant form, or insidious, developing over several months. In the acute phase, brucellosis presents as febrile illness associated with night sweats, malodorous perspiration, arthralgia, myalgia, and osteoarticular pain, particularly low back pain. Relapse is defined as reemergence of disease within 6 months of initial presentation, often owing to poor compliance with treatment, which thus leads to antibiotic resistance. In the chronic phase, the most common manifestations include persistent or cyclic low back pain and psychiatric symptoms. Complications of brucellosis are variable. Constitutional symptoms and osteoarticular disease are universally common, particularly sacroiliitis and spondylitis. Genitourinary complications, including epididymo-orchitis in males and pyelonephritis in females, are common. Neurological complications, including brain abscess, meningitis, meningoencephalitis, and encephalitis, occur in $5 \%-7 \%$ of reported cases. Hepatitis manifesting as transaminitis and hepatomegaly is frequently observed. Pulmonary complications are rare. Endocarditis is the most common complications of brucellosis leading to mortality. ${ }^{1-4}$

Diagnosis of brucellosis begins with a history and physical exam. A careful assessment of epidemiological risk factors is crucial. An assay of blood, bone marrow, or other body fluids or tissue culture is considered gold standard. However, it is imperative to underscore that Brucella culture requires a longer incubation period. There are multiple serological tests used to diagnose brucellosis. The most common serological test is serum agglutination test, where agglutination titers $\geq 1: 160$ considered diagnostic, although in endemic area a titer of $\geq 1: 320$ is considered diagnostic. Enzyme-linked immunosorbent assay (ELISA) is another popular serological method used to diagnose brucellosis. ELISA detects total Brucella immune-specific IgM, IgA, and IgG immunoglobulins. It is the preferred test for chronic and complicated cases, as well as for diagnosis of neurobrucellosis. Magnetic resonance imaging is the imaging modality of choice for vertebral and central nervous system assessment..$^{3-5}$

The preferred first-line treatment in adults with uncomplicated, nonlocalized disease consists of doxycycline-aminoglycoside combination. Streptomycin (Troge Medical GMBH, Hamburg, Germany) is typically used for the first 14-21 days; gentamicin is another option typically used for the first 10-14 days. The recommended duration of treatment is for 6 weeks. An alternative regimen includes doxycycline (Tabuk Pharmaceuticals Mfg. co., Tabuk, Saudi Arabia) plus rifampin (Sanofi Aventis, Bridgewater, NJ, USA) for a total of 6 weeks, but this regimen shows a higher failure rate and is not recommended for osteoarticular disease. ${ }^{6,7}$ The proposed therapy for brucellosis endocarditis by the European Society of Cardiology (ESC) includes doxycycline plus trimethoprim-sulfamethoxazole plus rifampin for 3-6 months, with some recommend adding gentamicin for the first 3 weeks. ${ }^{8}$ Cardiovascular implantable electronic device (CIED) infection due to brucellosis is rare and has been reported in few case study series. ${ }^{9-12}$

We report a case of $B$. melitensis infective endocarditis in a patient with CIED where, contrary to most authorities recommending removal of device, because of the presence of multiple comorbid conditions and age, an attempt was made to keep the device and place the patient on lifelong prophylaxis treatment.

\section{Case}

A 70-year-old Kuwaiti male with a known history of type 2 diabetes mellitus, hypertension, atrial fibrillation, and sick sinus syndrome status post pacemaker placement (placed approximately a year prior to his presentation) presented to our facility with a chief complaint of a mechanical fall, dizziness, urinary incontinence, intermittent subjective fever, and nausea with transient nonbilious, nonbloody emesis. The patient's travel history is remarkable for recent trip to 
Kingdom of Saudi Arabia, where patient acknowledged drinking unpasteurized camel milk.

On admission, the patient was febrile with a temperature of $38.9^{\circ} \mathrm{C}$. Hemodynamic status was acceptable, as was his respiratory status. A visual exam showed a sluggish, illlooking elderly male who looked older than his stated age. The physical exam was remarkable as it showed a new pansystolic murmur. No evidence of infective endocarditis stigmata was observed. No appreciable musculoskeletal ailment or joint pain with active and passive motion was noted. Initial lab tests showed no evidence of leukocytosis or leukopenia. White blood cell differential was unremarkable. Chemistry panel showed mild hyponatremia and hypoglycemia. Imaging studies included acute abdominal series and computed tomography of head, both of which were unremarkable. Two sets of blood cultures as well as a urine culture were performed on admission.

The hospital course was remarkable with persistent fever spikes for the first week. The urine culture yielded no growth. However, blood culture turned out positive in both the sets, showing $B$. melitensis 11 days after initial collection of sample. In light of these findings, the primary team started the patient on doxycycline and rifampin. Consultation with the Infectious Disease service was sought. Unfortunately, initial ELISA titers were not collected at the time of initiation of treatment. A transthoracic echo (TTE) was ordered (Figure 1). Results showed heavy calcification and thickening of aortic valve with two mobile masses attached to the aortic valve. One mass protruded into the left ventricular outflow tract with focal calcification measuring $13 \times 7 \mathrm{~mm}$; the second mass was at the level of the anterior

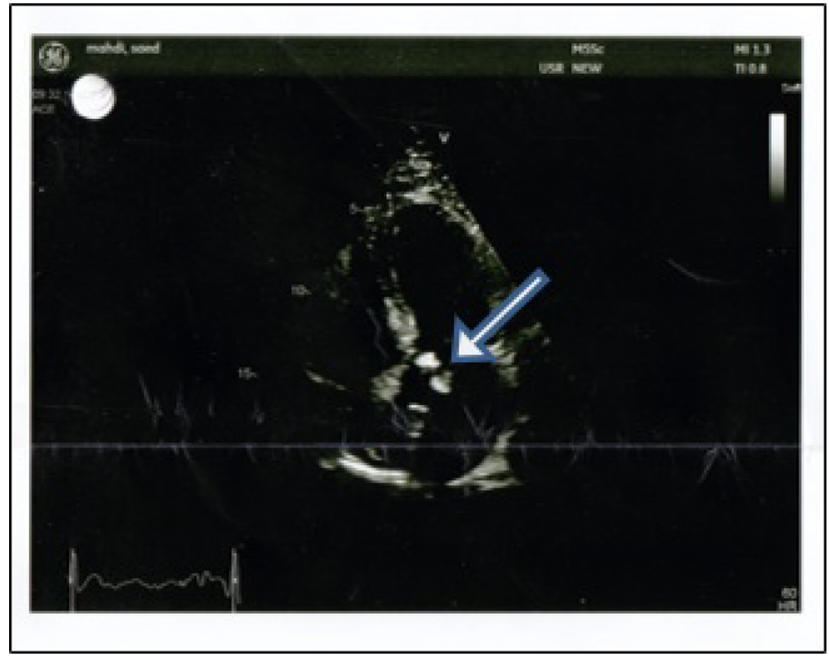

Figure I TTE shows heavy calcification and thickening of aortic valve with two mobile masses attached to the aortic valve. Abbreviation: TTE, transthoracic echo. mitral valve leaflet junction with the aortic valve, measuring $6 \times 3 \mathrm{~mm}$. In light of these findings and given the history of pacemaker placement, a transesophageal echo (TEE) was requested, which was performed several days later at a different specialized institution. The results showed no evidence of vegetation at the aortic valve or mitral valve. However, a thread mass was seen at the Eustachian valve position. The two pacing leads, one in right atrium appendage and the other in right valve apex, looked smooth and clean with no clear adherence. The discrepancy between TTE and TEE is likely due to poor quality of TTE for diagnosis of infective endocarditis. ${ }^{13}$

Recommendations by the cardiology team were for conservative treatment with antibiotics, with a plan to repeat TEE in 4 weeks. Cotrimoxazole (Julphar, Gulf Pharmaceutical Industries, Ras al-khaimah, UAE) and gentamicin were added to the treatment course by the Infectious Disease time, per the 2015 ESC Guidelines for the management of infective endocarditis. ${ }^{8}$ The plan was to treat with aminoglycoside for the first 3 weeks, and then downgrade to a three-drug regimen. A repeat blood culture a week into his treatment course showed sterilization of bloodstream. The patient's fever also abated several days into his treatment course. Unfortunately, 2 weeks into his four-drug regimen, the patient developed an oliguric acute kidney injury, which was attributed to aminoglycoside nephrotoxicity. Gentamicin was thus discontinued. Regrettably, in spite of aggressive treatment, the patient's renal failure worsened, and so he required several weeks of hemodialysis. The patient remained in the hospital during this period. He understandably became frustrated with the prolonged treatment course and unforeseen complications. He refused to repeat the echo. Two months into his hospital course, the kidney function gradually began to improve and hemodialysis was discontinued. Throughout this period, the patient remained on the three-drug regimen, rifampin, doxycycline, and cotrimoxazole. He remained afebrile throughout the remainder of his hospital course. The patient was subsequently discharged after completing a total of 3 months of antibiotic treatment.

At the time of discharge, the patient's Brucella titer was $<1: 160$. Unfortunately, the patient did not show up to either of his follow-up outpatient appointments. Two follow-up phone calls were made. The first phone call was made to inquire about his "no show" to the outpatient follow-up appointment. The patient stated that he was doing well, and did not deem follow-up as being necessary. The second phone call was made 2 months following his discharge, 5 months from the time of his initial admission to the hospital; the patient stated that he was doing well. He denied any fever, night sweats, myalgia, arthralgia, unusual rashes, palpitations, syncope, 
or chest pain. He continued to refuse any follow-up with medical professionals.

\section{Informed consent}

Written informed consent has been obtained from the patient to have the case details and any accompanying images published.

\section{Conclusion}

Universally, in all published studies on Brucella infective endocarditis with a cardiovascular implantable device, the recommendation was for device removal and extended treatment with doxycycline combined with rifampin and/or trimethoprim-sulfamethoxazole, plus parenteral aminoglycosides for the first 3 weeks. There are no specific guidelines regarding timing of new device reimplantation. Elmardi et $\mathrm{al}^{9}$ placed a new contralateral CIED on their two patients 3 months later. To the best of our knowledge, there have been two cases in the literature with brucellosis-infected CIED where an attempt was made to retain the device. In both cases, relapse occurred upon discontinuation of treatment. It is not clear whether lifelong antibiotic suppression treatment would prevent relapse. However, in frail elderly patients with multiple comorbidities, when device removal may be associated with increased risk and multiple complications, the benefits of suppressive therapy may outweighs the risk of device removal. ${ }^{14}$ It remains to be seen in the coming months and years whether our patient will continue to remain disease free. We will continue to periodically contact him and followup with him in the hope of maintaining close surveillance.

\section{Disclosure}

The authors report no conflicts of interest in this work.

\section{References}

1. Bennett JE, Dolin R, Blaser MJ. Mandell, Douglas, and Bennett's Principles and Practice of Infectious Diseases. 8th ed. Philadelphia, PA: Elsevier; 2015.

2. Corbel M. Brucellosis in Humans and Animals: FAO, OIE, WHO. Geneva:WHO; 2006. Available from: http://www.who.int/csr/resources/ publications/Brucellosis.pdf. Accessed June 5, 2017.

3. Pappas G, Akritidis N, Bosilkovski M, Tsianos E. Brucellosis. N Engl J Med. 2005;352(22):2325-2336.

4. Franco MP, Mulder M, Gilman RH, Smits HL. Human brucellosis. Lancet Infect Dis. 2007;7(12):775-786.

5. Kasper DL, Fauci AS, editors. Harrison's Infectious Diseases. 3rd ed. New York, NY: McGraw Hill; 2017.

6. Solís García del Pozo J, Solera J. Systematic review and meta-analysis of randomized clinical trials in the treatment of human brucellosis. PLoS One. 2012;7(2):e32090.

7. Al-Tawfiq JA, Memish ZA. Antibiotic susceptibility and treatment of brucellosis. Recent Pat Antiinfect Drug Discov. 2013;8(1):51-54.

8. Habib G, Lancellotti P, Antunes MJ, et al. 2015 ESC guidelines for the management of infective endocarditis: the task force for the management of infective endocarditis of the European Society of Cardiology (ESC). Endorsed by: European Association for Cardio-Thoracic Surgery (EACTS), the European Association of Nuclear Medicine (EANM). Eur Heart J. 2015;36(44):3075-3128.

9. Elmardi ME, Al Hebaishi Y, Al Kassab S, et al. Implantable cardioverter device infection due to Brucella melitensis in two patients. Hellenic $J$ Cardiol. Epub 2016 Aug 20. pii: S1109-9666(16)30150-6.

10. Dhand A, Ross JJ. Implantable cardioverter-defibrillator infection due to Brucella melitensis: case report and review of brucellosis of cardiac devices. Clin Infect Dis. 2007;44(4):e37-e39.

11. de la Fuente A, Sanchez JR, Uriz J, Reparaz J, Lopez-Coronado JL, Moriones I. Infection of a pacemaker by Brucella melitensis. Tex Heart Inst J. 1997;24(2):129-130.

12. Miragliotta G, Mosca A, Tantimonaco G, De Nittis R, Antonetti R, Di Taranto A. Relapsing brucellosis related to pacemaker infection. Ital Heart J. 2005;6(7):612-613.

13. Sekar P, Johnson JR, Thurn JR, et al. Comparative sensitivity of transthoracic and transesophageal echocardiography in diagnosis of infective endocarditis among veterans with staphylococcus aureus bacteremia. Open Forum Infect Dis. 2017;4(2):ofx035.

14. Tan EM, DeSimone DC, Sohail MR, et al. Outcomes in patients with cardiovascular implantable electronic device infection managed with chronic antibiotic suppression. Clin Infect Dis. 2017;64(11): $1516-1521$.
Infection and Drug Resistance

Publish your work in this journal

Infection and Drug Resistance is an international, peer-reviewed openaccess journal that focuses on the optimal treatment of infection (bacterial, fungal and viral) and the development and institution of preventive strategies to minimize the development and spread of resistance. The journal is specifically concerned with the epidemiology of antibiotic

\section{Dovepress}

resistance and the mechanisms of resistance development and diffusion in both hospitals and the community. The manuscript management system is completely online and includes a very quick and fair peerreview system, which is all easy to use. Visit http://www.dovepress.com/ testimonials.php to read real quotes from published authors. 